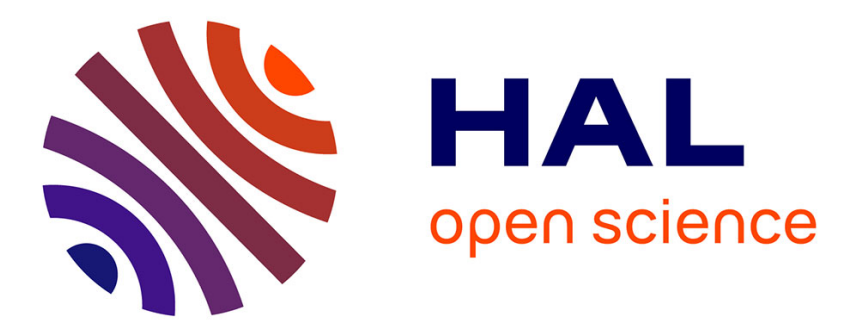

\title{
On the Coupling of 3D and 1D Navier-Stokes Equations for Flow Problems in Compliant Vessels
}

Luca Formaggia, Jean-Frédéric Gerbeau, Fabio Nobile, Alfio Quarteroni

\section{To cite this version:}

Luca Formaggia, Jean-Frédéric Gerbeau, Fabio Nobile, Alfio Quarteroni. On the Coupling of 3D and 1D Navier-Stokes Equations for Flow Problems in Compliant Vessels. [Research Report] RR-3862, INRIA. 2000. inria-00072794

\section{HAL Id: inria-00072794 \\ https://hal.inria.fr/inria-00072794}

Submitted on 24 May 2006

HAL is a multi-disciplinary open access archive for the deposit and dissemination of scientific research documents, whether they are published or not. The documents may come from teaching and research institutions in France or abroad, or from public or private research centers.
L'archive ouverte pluridisciplinaire HAL, est destinée au dépôt et à la diffusion de documents scientifiques de niveau recherche, publiés ou non, émanant des établissements d'enseignement et de recherche français ou étrangers, des laboratoires publics ou privés. 


\section{On the Coupling of $3 D$ and $1 D$ Navier-Stokes Equations for Flow Problems in Compliant Vessels}

Luca Formaggia — Jean-Frédéric Gerbeau — Fabio Nobile — Alfio Quarteroni

\section{$\mathbf{N}^{\circ} 3862$}

Janvier 2000

THÈME 4 



\title{
On the Coupling of $3 \mathrm{D}$ and $1 \mathrm{D}$ Navier-Stokes Equations for Flow Problems in Compliant Vessels
}

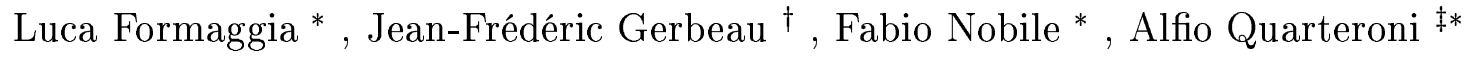 \\ Thème 4 - Simulation et optimisation \\ de systèmes complexes \\ Projet M3N
}

Rapport de recherche $\mathrm{n}^{\circ} 3862$ - Janvier $2000-25$ pages

\begin{abstract}
For the analysis of flows in compliant vessels, we propose an approach to couple the original 3D equations with a convenient 1D model. This multiscale strategy allows for a dramatic reduction of the computational complexity and is suitable for "absorbing" outgoing pressure waves. In particular, it is of utmost interest for the description of blood motion in the arterial system.
\end{abstract}

Key-words: fluid structure interaction, multi-scale coupling, hemodynamics

* Département de Mathématiques, École Polytechnique Fédérale, CH-1015, Lausanne, SWITZERLAND, email: Luca.Formaggia@epfl.ch, Fabio.Nobile@epfl.ch, Alfio.Quarteroni@epfl.ch

$\dagger$ INRIA Rocquencourt - Projet M3N - email: Jean-Frederic.Gerbeau@inria.fr

$\ddagger$ Dipartimento di Matematica "F. Brioschi", Politecnico di Milano, Piazza Leonardo da Vinci 32, I-20133 Milano, ITALY 


\section{Couplage 3D/1D des équations de Navier-Stokes dans des vaisseaux déformables}

Résumé : Afin d'analyser les écoulements dans des vaisseaux déformables, nous proposons une approche pour coupler les équations tridimensionnelles originales à un modèle monodimensionnel. Cette stratégie multi-échelle réduit considérablement la complexité du calcul et permet d'"absorber" les ondes de pression sortantes. L'étude s'applique en particulier à la description de l'écoulement sanguin dans le système artériel.

Mots-clés : interaction fluide structure, couplage multi-échelle, hémodynamique 


\section{Introduction}

The study of a transient incompressible flow inside a compliant vessel, that is a vessel whose walls deform under the action of the fluid, is of considerable interest, for instance in the simulation of blood flow in large arteries ([13]).

The mathematical problem consists of the $2 \mathrm{D}$ or 3D Navier-Stokes equations describing the fluid motion, coupled with a suitable model that describes the displacement of the vessel wall (hereafter called "the structure"). We will refer to this coupled system as the 2D/3D fluid-structure model.

This problem is interesting from the mathematical and numerical modelling side, as it exhibits some peculiar features like the appearance of travelling pressure waves along the vessel. Indeed, even if the flow is governed by parabolic equations such as the incompressible Navier-Stokes, the behaviour of the coupled fluid-structure system is for many aspects more akin to that of a hyperbolic problem.

As a consequence, an additional complexity arises in the treatment of the "inflow" and "outflow" boundaries, where one would like to have a correct representation of the travelling waves, without spurious reflections. In this work, this issue has been tackled by coupling the $2 \mathrm{D} / 3 \mathrm{D}$ fluid-structure problem with a reduced one-dimensional model, which acts as an "absorbing" device for the waves exiting the computational domain.

This reduced model is obtained integrating the fluid equations over each section normal to the axis of the vessel and describes the coupled system in terms of transversally averaged flow rate and pressure.

This work is motivated also by the prospect of implementing multi-scale models for the human cardiovascular system. It is clear that a realistic detailed numerical simulation of the flow in a segment of an artery (like, for instance, the carotid bifurcation or a trait of a coronary) may not be fully accomplished without accounting for the interactions with the remaining part of the cardiovascular system. One possibility to that direction is offered by the coupling of the detailed 2D/3D simulation with a simplified 1D or even lumped parameter modelisation of the rest of the global system. The work detailed in this paper represents a necessary step towards that goal.

In section 1 we will derive the formulation for the 3D fluid-structure model. A simplified onedimensional formulation for the structure is also presented and used for the stability analysis of the coupled problem. The main result of the section is an energy inequality which, under a condition on the positivity of the kinetic energy flux at the outflow, provides a bound of the global energy of the coupled problem in terms of initial and boundary data.

The section 2 is devoted to the derivation and analysis of the 1D model of the flow inside a compliant vessel. Under suitable approximations, we derive a hyperbolic system of equations with source term. A proper set of boundary conditions is derived by means of standard characteristics analysis. Then the stability of the system is analysed for the sub-critical case, which is the most relevant for our target applications. Again a bound on the energy of the system is found in terms of the initial and boundary data, stating the stability of the 1D model.

Next, we present several strategies for coupling the two models and possible algorithms based on sub-domain iterations. Details on the adopted numerical discretisation are given in section 4 . For the 2D/3D fluid-structure models we have employed finite element techniques and we have used an Arbitrary Lagrangian Eulerian formulation to account for the boundary movement in the fluid region. The 1D hyperbolic system is solved by a Lax-Wendroff scheme.

Finally, numerical results are provided both for the 3D-1D and the 2D-1D models coupling. We compare the solution behaviour when a homogeneous Neumann boundary condition on the velocity is prescribed at the outlet with the solution obtained by the coupling. The latter shows a greatly reduced level of spurious reflections. For the $2 \mathrm{D}-1 \mathrm{D}$ case a quantitative analysis on the error is given by comparing the results with that obtained on a vessel of double length. 


\section{The 3D model}

In this section and in the next one, we illustrate the models we have used to describe the fluid and the structure movement and their interaction: the 3D fluid structure interaction problem and the derivation of the $1 \mathrm{D}$ reduced model, respectively.

Let us consider the district illustrated in Fig. 1, which we will denote by $\Omega$. The vessel has a

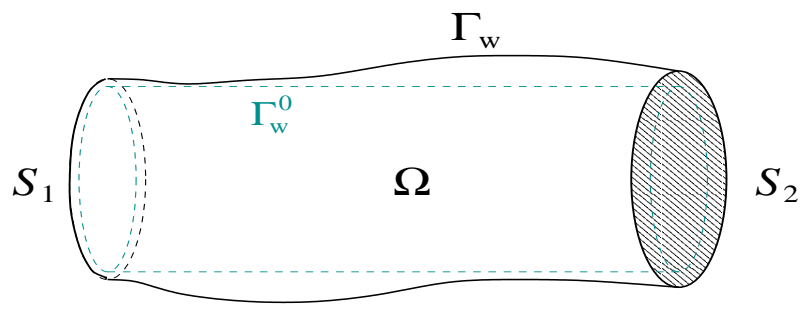

Figure 1: Simple compliant tube.

compliant wall denoted by $\Gamma_{w}$. The boundary of the vessel is completed by the sections $\mathcal{S}_{1}$ and $\mathcal{S}_{2}$, connecting the district to the rest of the system. More precisely, $\mathcal{S}_{1}$ is the upstream section; $\mathcal{S}_{2}$ is the downstream section, through which the fluid enters and leaves $\Omega$ respectively.

We will denote by $\mathbf{u}(t, \mathbf{x})$ (with $\mathbf{x} \in \Omega, t>0$ ) the fluid velocity field, by $p(t, \mathbf{x}$ ) the pressure and by $\rho$ the (constant) fluid density. We describe the fluid motion through the classical Navier-Stokes equations

$$
\left\{\begin{array}{l}
\partial_{t} \mathbf{u}+\mathbf{u} \cdot \nabla \mathbf{u}-\frac{1}{\rho} \operatorname{div} \mathbf{T}(\mathbf{u}, p)=0 \\
\operatorname{div} \mathbf{u}=0
\end{array} \quad \text { in } \Omega,\right.
$$

where $\mathbf{T}$ is the Cauchy stress tensor. Hereafter, we will consider the case of Newtonian fluids where we can use the following constitutive relation

$$
\mathbf{T}(\mathbf{u}, p)=-p \mathbf{I}+\mu \nabla \mathbf{u},
$$

$\mu$ being the fluid viscosity.

Remark 1.1 Concerning the blood flow problem, in large arteries blood can be assumed to behave as a Newtonian fluid ([10, 21]) while in small vessels and capillaries, its rheology is more complex. An account in given in [2, 12, 21].

Whatever PDE model is considered for the vessel wall, it would provide the position of every point on $\Gamma_{w}$ at any time $t>0$, which is typically described by means of the displacement $\boldsymbol{\eta}$ with respect to a reference position $\Gamma_{w}^{0}$ (see for instance $[1,8]$ ).

Then, matching conditions at $\Gamma_{w}$ may be provided as follows:

$$
\left\{\begin{array}{l}
\mathbf{u}=\dot{\boldsymbol{\eta}} \\
-\mathbf{T} \cdot \mathbf{n}-p_{\text {ext }} \mathbf{n}=\mathbf{\Phi}
\end{array} \quad \text { on } \Gamma_{w},\right.
$$

where $p_{\text {ext }}$ is a given external pressure, $\boldsymbol{\Phi}$ is the forcing term acting on the wall and $\mathbf{n}$ is the outward unit vector to $\Gamma_{w}$.

The first of (2) guarantees the perfect adherence of the fluid to the structure while the second one states the continuity of the stresses at the interface (according to the action and reaction principle).

Both fluid and structure equations must be supplied with initial conditions (resp. on $\Omega$ and $\Gamma_{w}^{0}$ ) and boundary conditions (resp. on $\mathcal{S}_{1}, \mathcal{S}_{2}$ and $\partial \mathcal{S}_{1}, \partial \mathcal{S}_{2}$ ).

About the structure model, we will address two cases: 
1. A shell model, i.e. a 3D structure in which one dimension, the thickness, is much lower than the other ones. In particular we have considered the so-called geometrically exact shell model developed in $[16,17,18]$. The main unknowns are the mid-surface position of the shell and its normal (or more precisely a director vector) at each point. In this model, deformations are described without approximation on the geometry - even when large displacements are considered - which is particularly interesting for the fluid-structure interaction problem arising in the study of blood flow.

2. A simplified structural model, which is derived for a cylindrical configuration (generalised string model; see [13]). Let

$$
\Gamma_{w}^{0}=\left\{(r, \theta, z): \quad r=R_{0}, 0 \leq z \leq L, 0 \leq \theta<2 \pi\right\}
$$

be a cylindrical reference surface of radius $R_{0}$; we neglect the longitudinal and angular displacement while the radial displacement $\eta_{r}=\eta_{r}(t, \theta, z)$ is given by

$$
\rho_{w} h \frac{\partial^{2} \eta_{r}}{\partial t^{2}}-k G h \frac{\partial^{2} \eta_{r}}{\partial z^{2}}+\frac{E h}{1-\nu^{2}} \frac{\eta_{r}}{R_{0}^{2}}-\gamma \frac{\partial^{3} \eta_{r}}{\partial z^{2} \partial t}=f(t, \theta, z)
$$

Here, $h$ is the wall thickness, $R_{0}$ is the arterial reference radius at rest; $k$ is the so called Timoshenko shear correction factor; $G$ the shear modulus; $E$ the Young modulus, $\nu$ the Poisson ratio (which for an incompressible material is equal to $1 / 2) ; \rho_{w}$ the wall volumetric mass, $\gamma$ is a viscoelastic parameter and, finally $f$ is an external forcing term.

Model (3) is basically derived from the equations of linear elasticity for a cylindrical tube with small thickness, under the hypotheses of plain stresses and membrane deformations (i.e negligible elastic bending terms). The term $k G h \frac{\partial^{2} \eta_{r}}{\partial z^{2}}$ accounts for shear deformations ([15]) while the term $\gamma \frac{\partial^{3} \eta_{r}}{\partial z^{2} \partial t}$ introduces a viscoelastic behaviour.

For the purpose of the mathematical analysis, we will play with a $2 \mathrm{D}$ model obtained by intersecting the tube $\Omega$ with a plane $\theta=\bar{\theta}$ (see fig. 2). Correspondingly, we will consider the $2 \mathrm{D}$ problem arising form the combination of the $2 \mathrm{D}$ Navier-Stokes equations for the fluid with eq. (3) to describe the motion of the upper and lower boundary (with a frozen value of the angle $\theta$ ).
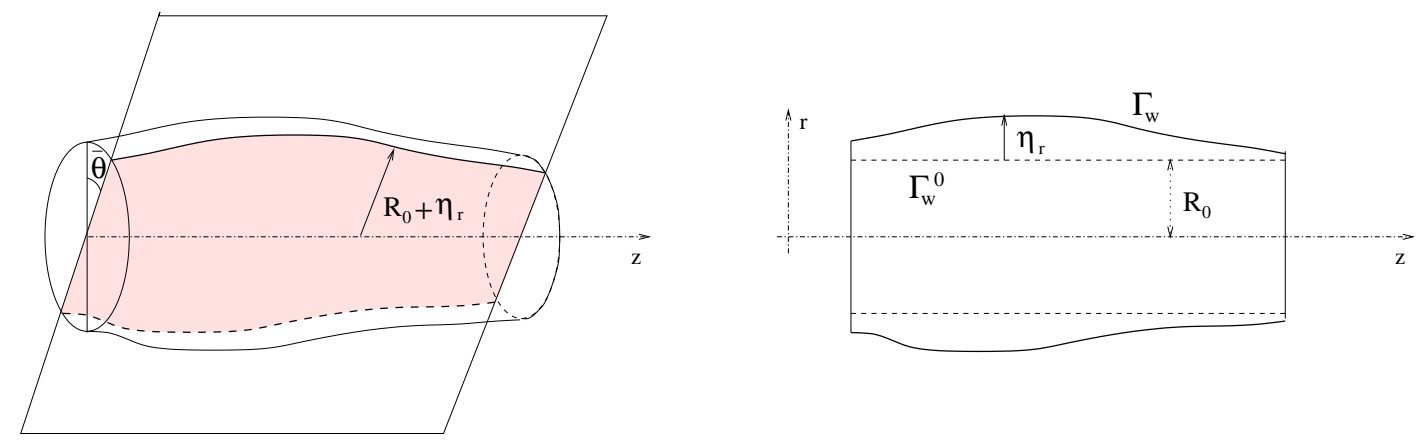

Figure 2: Intersecting the cylinder with the plane $\theta=\bar{\theta}$ we obtain a $2 \mathrm{D}$ geometry with a $1 \mathrm{D}$ compliant wall

Remark 1.2 Although not being completely realistic for blood flow problems, this simplified $2 D$ analysis maintains all the mathematical aspects peculiar to the original coupled fluidstructure problem and will therefore be adopted to test the numerical coupling algorithms we will present in Section 4. 


\subsection{Energy inequality for the coupled fluid-structure problem}

Let us consider the 3D Navier-Stokes equations coupled with the structure model (3) through the matching conditions (2). In eq. (3), the forcing term $f(t, \theta, z)$ is given by the radial component $\Phi_{r}$ of the normal stress exerted by the fluid, recast in the reference configuration $\Gamma_{w}^{0}$, that is

$$
f(t, \theta, z)=\Phi_{r} \frac{R}{R_{0}} \sqrt{1+\left(\frac{\partial \eta_{r}}{\partial z}\right)^{2}+\left(\frac{1}{R} \frac{\partial \eta_{r}}{\partial \theta}\right)^{2}} \quad \text { on } \Gamma_{w}^{0} .
$$

Here we have noted $R=R_{0}+\eta_{r}$, while the term under square root accounts for the change in the surface measure passing from $\Gamma_{w}$ to $\Gamma_{w}^{0}$. Clearly, with such a right hand side, eq. (3) becomes non-linear and difficult to handle with. However, as we will show in sec. 4 , at the numerical level we will decouple the fluid and structure problems, and the right hand side will be computed at a previous step of the staggered algorithm.

Remark 1.3 Since, from (2), we have $\Phi_{r}=-\left(\mathbf{T}+p_{\text {ext }} \mathbf{I}\right) \cdot \mathbf{n} \cdot \mathbf{e}_{r}$ where $\mathbf{T}$ is the Cauchy stress tensor field, we may note that

$$
\mathbf{n}=\frac{\mathbf{N}}{|\mathbf{N}|} \quad \text { with } \mathbf{N}=R \mathbf{e}_{r}-\frac{\partial \eta_{r}}{\partial \theta} \mathbf{e}_{\theta}-R \frac{\partial \eta_{r}}{\partial z} \mathbf{e}_{z}
$$

and the right hand side of (3) becomes

$$
\begin{aligned}
\Phi_{r} \frac{R}{R_{0}} \sqrt{1+\left(\frac{\partial \eta_{r}}{\partial z}\right)^{2}+\left(\frac{1}{R} \frac{\partial \eta_{r}}{\partial \theta}\right)^{2}}=-\frac{1}{R_{0}}\left(\mathbf{T}+p_{\text {ext }} \mathbf{I}\right) \cdot \mathbf{N} \cdot \mathbf{e}_{r}= \\
\quad\left(-T_{r r}-p_{e x t}+T_{r \theta} \frac{1}{R} \frac{\partial \eta_{r}}{\partial \theta}+T_{r z} \frac{\partial \eta_{r}}{\partial z}\right) \frac{R}{R_{0}}
\end{aligned}
$$

which is now linear in $\eta_{r}$ provided we approximate $R$ by $R_{0}$. Moreover, under the same approximation, if $\mathbf{T}$ is an isotropic tensor field, i.e. $\mathbf{T}=-p \mathbf{I}$, we have $\left(-\mathbf{T}-p_{\text {ext }} \mathbf{I}\right) \cdot \mathbf{N} \cdot \mathbf{e}_{r} / R_{0}=p-p_{\text {ext }}$ and the right hand side reduces to the only pressure difference $p-p_{\text {ext }}$.

We now analyse in more detail the coupled fluid-structure problem with the following initial and boundary conditions:

for the fluid

$$
\begin{array}{ll}
\mathbf{u}=\mathbf{u}_{0} & \text { for } t=0, \text { in } \Omega \\
\mathbf{u}=0 & \text { on } \mathcal{S}_{1} \\
\mathbf{T} \cdot \mathbf{n}=0 & \text { on } \mathcal{S}_{2}
\end{array}
$$

for the structure

$$
\begin{array}{ll}
\eta_{r}=\eta_{0}, \dot{\eta}_{r} \mathbf{e}_{r}=\mathbf{u}_{0} & \text { for } t=0, \text { on } \Gamma_{w} \\
\eta_{r}=0 & \text { for } z=0, z=L
\end{array}
$$

For the sake of simplicity, and without loss of generality, we will consider only the case $p_{\text {ext }}=0$. Let us rewrite eq. (3) in the general form

$$
\tilde{\rho}_{w} \frac{\partial^{2} \eta_{r}}{\partial t^{2}}-a \frac{\partial^{2} \eta_{r}}{\partial z^{2}}+b \eta_{r}-c \frac{\partial^{3} \eta_{r}}{\partial^{2} z \partial t}=\Phi_{r} \frac{R}{R_{0}} \sqrt{1+\left(\frac{\partial \eta_{r}}{\partial z}\right)^{2}+\left(\frac{1}{R} \frac{\partial \eta_{r}}{\partial \theta}\right)^{2}}
$$

on $\Gamma_{w}^{0}$ and for $t>0$, where all the coefficients $\tilde{\rho}_{w}, a, b$, and $c$ are positive quantities. We wish to derive an energy inequality for the coupled problem. The following Lemma holds 
Lemma 1.1 The coupled problem (1), (7) with matching conditions (2) and initial and boundary conditions (5) and (6) satisfies the following energy equality

$$
\begin{aligned}
\frac{1}{2} \frac{d}{d t}\left\{\rho\|\mathbf{u}\|_{L^{2}(\Omega)}^{2}+\tilde{\rho}_{w}\left\|\frac{\partial \eta_{r}}{\partial t}\right\|_{L^{2}\left(\Gamma_{w}^{0}\right)}^{2}+a\left\|\frac{\partial \eta_{r}}{\partial z}\right\|_{L^{2}\left(\Gamma_{w}^{0}\right)}^{2}+b\left\|\eta_{r}\right\|_{L^{2}\left(\Gamma_{w}^{0}\right)}^{2}\right\}+ \\
\mu\|\nabla \mathbf{u}\|_{L^{2}(\Omega)}^{2}+c\left\|\frac{\partial^{2} \eta_{r}}{\partial z \partial t}\right\|_{L^{2}\left(\Gamma_{w}^{0}\right)}^{2}+\frac{1}{2} \rho \int_{\mathcal{S}_{2}}|\mathbf{u}|^{2} \mathbf{u} \cdot \mathbf{n} d \gamma=0
\end{aligned}
$$

Moreover, if

$$
\int_{\mathcal{S}_{2}}|\mathbf{u}|^{2} \mathbf{u} \cdot \mathbf{n} d \gamma>0, \quad \forall t>0
$$

we obtain the a-priori energy estimate

$$
\begin{aligned}
\rho\|\mathbf{u}(T)\|_{L^{2}(\Omega)}^{2}+\tilde{\rho}_{w}\left\|\frac{\partial \eta_{r}}{\partial t}(T)\right\|_{L^{2}\left(\Gamma_{w}^{0}\right)}^{2}+ & a\left\|\frac{\partial \eta_{r}}{\partial z}(T)\right\|_{L^{2}\left(\Gamma_{w}^{0}\right)}^{2}+b\left\|\eta_{r}(T)\right\|_{L^{2}\left(\Gamma_{w}^{0}\right)}^{2}+ \\
& 2 \int_{0}^{T} \mu\|\nabla \mathbf{u}\|_{L^{2}(\Omega)}^{2} d t+2 \int_{0}^{T} c\left\|\frac{\partial^{2} \eta_{r}}{\partial z \partial t}\right\|_{L^{2}\left(\Gamma_{w}^{0}\right)}^{2} d t \leq C,
\end{aligned}
$$

where $C$ is a constant which depends on the initial conditions $\mathbf{u}_{0}$ and $\eta_{0}$.

\section{Proof.}

We first multiply eq. (7) by $\frac{\partial \eta_{r}}{\partial t}$ and integrate on the cylindrical surface $\Gamma_{w}^{0}$, obtaining

$$
\begin{aligned}
& \frac{1}{2} \frac{d}{d t}\left\{\tilde{\rho}_{w}\left\|\frac{\partial \eta_{r}}{\partial t}\right\|_{L^{2}\left(\Gamma_{w}^{0}\right)}^{2}+a\left\|\frac{\partial \eta_{r}}{\partial z}\right\|_{L^{2}\left(\Gamma_{w}^{0}\right)}^{2}+b\left\|\eta_{r}\right\|_{L^{2}\left(\Gamma_{w}^{0}\right)}^{2}\right\}+ \\
& c\left\|\frac{\partial^{2} \eta_{r}}{\partial z \partial t}\right\|_{L^{2}\left(\Gamma_{w}^{0}\right)}^{2}-\left.\left(a \frac{\partial \eta_{r}}{\partial z} \frac{\partial \eta_{r}}{\partial t}+c \frac{\partial^{2} \eta_{r}}{\partial z \partial t} \frac{\partial \eta_{r}}{\partial t}\right)\right|_{z=0} ^{z=L}= \\
& \int_{\Gamma_{w}^{0}} \Phi_{r} \frac{R}{R_{0}} \sqrt{1+\left(\frac{\partial \eta_{r}}{\partial z}\right)^{2}+\left(\frac{1}{R} \frac{\partial \eta_{r}}{\partial \theta}\right)^{2}} \frac{\partial \eta_{r}}{\partial t} R_{0} d \theta d z
\end{aligned}
$$

Thanks to the specific boundary conditions, the last term on the left side is identically zero, while the right hand side, if computed on the actual configuration $\Gamma_{w}$, becomes

$$
\int_{\Gamma_{w}^{0}} \Phi_{r} \sqrt{1+\left(\frac{\partial \eta_{r}}{\partial z}\right)^{2}+\left(\frac{1}{R} \frac{\partial \eta_{r}}{\partial \theta}\right)^{2}} \frac{\partial \eta_{r}}{\partial t} R d \theta d z=\int_{\Gamma_{w}} \Phi_{r} \frac{\partial \eta_{r}}{\partial t} d \gamma=\int_{\Gamma_{w}}(-\mathbf{T} \cdot \mathbf{n}) \cdot \mathbf{u} d \gamma
$$

where in the last equality we have exploited the coupling conditions (2). Then, the following energy equality holds for the structure problem

$$
\begin{aligned}
\frac{1}{2} \frac{d}{d t}\left\{\tilde{\rho}_{w}\left\|\frac{\partial \eta_{r}}{\partial t}\right\|_{L^{2}\left(\Gamma_{w}^{0}\right)}^{2}+a\left\|\frac{\partial \eta_{r}}{\partial z}\right\|_{L^{2}\left(\Gamma_{w}^{0}\right)}^{2}+b\left\|\eta_{r}\right\|_{L^{2}\left(\Gamma_{w}^{0}\right)}^{2}\right\}+ \\
\qquad\|\| \frac{\partial^{2} \eta_{r}}{\partial z \partial t} \|_{L^{2}\left(\Gamma_{w}^{0}\right)}^{2}=\int_{\Gamma_{w}}(-\mathbf{T} \cdot \mathbf{n}) \cdot \mathbf{u} d \gamma
\end{aligned}
$$

Analogously for the fluid equations, by multiplying the first of (1) by $\mathbf{u}$ and integrating over $\Omega$ we can derive the following energy estimate (see e.g. [13])

$$
\frac{1}{2} \rho \frac{d}{d t}\|\mathbf{u}\|_{L^{2}(\Omega)}^{2}+\mu\|\nabla \mathbf{u}\|_{L^{2}(\Omega)}^{2}-\int_{\mathcal{S}_{1} \cup \mathcal{S}_{2}}\left(\mathbf{T} \cdot \mathbf{n}-\frac{1}{2} \rho|\mathbf{u}|^{2} \mathbf{n}\right) \cdot \mathbf{u} d \gamma=\int_{\Gamma_{w}}(\mathbf{T} \cdot \mathbf{n}) \cdot \mathbf{u} d \gamma
$$

and, exploiting the boundary conditions, we have 


$$
\frac{1}{2} \rho \frac{d}{d t}\|\mathbf{u}\|_{L^{2}(\Omega)}^{2}+\mu\|\nabla \mathbf{u}\|_{L^{2}(\Omega)}^{2}+\int_{\mathcal{S}_{2}} \frac{1}{2} \rho|\mathbf{u}|^{2} \mathbf{n} \cdot \mathbf{u} d \gamma=\int_{\Gamma_{w}}(\mathbf{T} \cdot \mathbf{n}) \cdot \mathbf{u} d \gamma
$$

By summing equalities (12) and (13) the term $\int_{\Gamma_{w}}(\mathbf{T} \cdot \mathbf{n}) \cdot \mathbf{u} d \gamma$ cancels out and we obtain (8). Finally, integrating (8) in time between 0 and $T$, under the hypothesis (9), we obtain the desired inequality $(10) . \diamond$

The hypothesis (9) is actually satisfied if $\mathcal{S}_{2}$ is an outflow section, i.e. $\mathbf{u} \cdot \mathbf{n}>0$ for all $\mathbf{x} \in \mathcal{S}_{2}$. However, in vascular problems, this assumption is seldom true because the pulsating nature of blood flow might induce a flow reversal along portions of an artery during the cardiac beat.

We may observe that the "viscoelastic term" in (3) allows to obtain the appropriate regularity of the velocity field $\mathbf{u}$ on the boundary (see [13]).

In the derivation of energy inequality (10), we have considered homogeneous boundary conditions both for the fluid and the structure. However, the conditions $\eta=0$ at $z=0$ and $z=L$, which correspond to hold the wall ends fixed, are not realistic in the blood flow context. Since the model (3) for the structure is of propagative type, first order absorbing boundary conditions are a better choice, i.e.

$$
\begin{array}{ll}
\frac{\partial \eta_{r}}{\partial t}-\sqrt{\frac{a}{\tilde{\rho}_{w}}} \frac{\partial \eta_{r}}{\partial z}=0 & \text { at } z=0 \\
\frac{\partial \eta_{r}}{\partial t}+\sqrt{\frac{a}{\tilde{\rho}_{w}}} \frac{\partial \eta_{r}}{\partial z}=0 & \text { at } z=L .
\end{array}
$$

In this case, the final estimate (10) is still valid. Indeed the boundary term which appear in (11) now should read

$$
\begin{aligned}
-\left.\left(a \frac{\partial \eta_{r}}{\partial z} \frac{\partial \eta_{r}}{\partial t}+c \frac{\partial^{2} \eta_{r}}{\partial z \partial t} \frac{\partial \eta_{r}}{\partial t}\right)\right|_{z=0} ^{z=L}= \\
\quad \sqrt{\tilde{\rho}_{w} a}\left[\left(\left.\frac{\partial \eta_{r}}{\partial t}\right|_{z=0}\right)^{2}+\left(\left.\frac{\partial \eta_{r}}{\partial t}\right|_{z=L}\right)^{2}\right]+\frac{c}{2} \sqrt{\frac{\tilde{\rho}_{w}}{a}} \frac{d}{d t}\left[\left(\left.\frac{\partial \eta_{r}}{\partial t}\right|_{z=0}\right)^{2}+\left(\left.\frac{\partial \eta_{r}}{\partial t}\right|_{z=L}\right)^{2}\right] .
\end{aligned}
$$

This term, integrated in time, would eventually appear on the left hand side of inequality (10). We may note, however, that we obtain at both $z=0$ and $z=L$ the following expression

$$
\sqrt{\tilde{\rho}_{w} a} \int_{0}^{T}\left(\frac{\partial \eta_{r}}{\partial t}(\tau)\right)^{2} d \tau+\frac{c}{2} \sqrt{\frac{\tilde{\rho}_{w}}{a}}\left(\frac{\partial \eta_{r}}{\partial t}(T)\right)^{2}=\frac{c}{2} \sqrt{\frac{\tilde{\rho}_{w}}{a}}\left(\frac{\partial \eta_{r}}{\partial t}(0)\right)^{2} .
$$

Therefore, this additional term is positive and it depends only on the initial conditions.

Yet, conditions (14) and (15) are not compatible with the homogeneous Dirichlet boundary conditions for the fluid; indeed, if $\left.\eta\right|_{z=0} \neq 0$ and $\mathbf{u}=0$ on $\mathcal{S}_{1}$, the trace of $\mathbf{u}(t, x)$ on the boundary is discontinuous and thus not compatible with the regularity required on the solution of (1) (see. e.g. [14]).

A possible remedy consists in changing the condition $\mathbf{u}=0$ on $\mathcal{S}_{1}$ into

$$
\begin{aligned}
& \mathbf{u} \cdot \mathbf{e}_{z}=g \\
& \mathbf{T} \cdot \mathbf{n}-(\mathbf{T} \cdot \mathbf{n}) \cdot \mathbf{e}_{z}=0 \quad \text { on } \mathcal{S}_{1}
\end{aligned}
$$

with $g=0$ on $\partial \mathcal{S}_{1}$. An energy inequality for the coupled problem can be derived also in this case with standard calculations, taking a suitable harmonic extension $\tilde{g}$ of the non homogeneous data $g$ such that

$$
\left.\tilde{g}\right|_{\mathcal{S}_{1}}=g \quad \text { and }\left.\quad \tilde{g}\right|_{\Gamma_{w}}=0 .
$$

The calculations are here omitted for the sake of brevity. 


\section{The 1D model}

In the case where the boundary $\Gamma_{w}^{0}$ of the reference configuration $\Omega_{0}$ is a cylinder of radius $R_{0}$, a simplified 1D model can be obtained integrating, at each time $t>0$, the Navier-Stokes equations (1) over each section $\mathcal{S}(t, z)$ normal to the axis $z$ of the cylinder. In the sequel, $A(t, z)$ denotes the area of $\mathcal{S}(t, z)$ and $u_{z}(t, z)$ the axial velocity, while $Q(t, z)$ and $\bar{p}(t, z)$ are the flow rate (or flux) and the mean pressure in every section, given by

$$
Q(t, z)=\int_{\mathcal{S}(t, z)} u_{z}(t, z) d \sigma, \quad \bar{p}(t, z)=\frac{1}{A(t, z)} \int_{\mathcal{S}(t, z)} p(t, z) d \sigma .
$$

Finally $\bar{u}=Q / A$ denotes the mean axial velocity.

The 1D model which is obtained reads (see [4])

$$
\left\{\begin{array}{l}
\frac{\partial A}{\partial t}+\frac{\partial Q}{\partial z}=0 \\
\frac{\partial Q}{\partial t}+\frac{\partial}{\partial z}\left(\alpha \frac{Q^{2}}{A}\right)+\frac{A}{\rho} \frac{\partial \bar{p}}{\partial z}+K_{R} \frac{Q}{A}=0
\end{array}\right.
$$

The constant $K_{R}$ is a resistance parameter which accounts for fluid viscosity, while $\alpha$, sometimes indicated Coriolis coefficient, is defined as

$$
\alpha=A\left(\int_{\mathcal{S}} u_{z}^{2} d \sigma\right)\left(\int_{\mathcal{S}} u_{z} d \sigma\right)^{-2} .
$$

and accounts for the fact that the flux of momentum computed with the averaged quantities (i.e. $Q^{2} / A$ ) is in general different from the actual one. Even if, in general, $\alpha$ is function of $z$ and $t$, we have taken it constant and equal to one, which corresponds to assume a flat velocity profile.

System (17) is a system of two equations in three unknowns $(\bar{p}, A$ and $Q)$. For its closure, a third equation is provided by a suitable wall model relating the radial displacement (and henceforth the area $A$ ) to the mean pressure $\bar{p}$.

Here we consider a simple algebraic relation between pressure and area of the type

$$
\bar{p}=p_{\text {ext }}+\psi(A), \quad \text { with } \frac{d \psi}{d A}>0 \quad \text { and } \quad \psi\left(A_{0}\right)=0,
$$

where $p_{\text {ext }}$ is a constant external pressure and $A_{0}=\pi R_{0}^{2}$ is the reference area. Then, system (17) turns out to be hyperbolic and possesses two distinct eigenvalues

$$
\lambda_{1,2}=\bar{u} \pm c_{1}, \quad \text { where } c_{1}^{2}(A)=\frac{A}{\rho} \frac{\partial \psi(A)}{\partial A} .
$$

The corresponding eigenfunctions are the characteristic variable, here given by

$$
W_{1,2}=\bar{u} \pm \int_{A_{0}}^{A} \frac{c_{1}(\tau)}{\tau} d \tau
$$

They are not constant along the characteristic curves $d z_{i} / d t=\lambda_{i}, i=1,2$ because of the presence of a source term.

An example of algebraic relation of type (18) can be derived considering the static counterpart of equation (3), neglecting the spatial derivative and considering the pressure jump $\bar{p}-p_{\text {ext }}$ as forcing term. In such a case, we have

$$
\bar{p}-p_{e x t}=\frac{E h}{1-\nu^{2}} \frac{\eta_{r}}{R_{0}^{2}} .
$$


Since $A=\pi\left(R_{0}+\eta_{r}\right)^{2}$, we obtain

$$
\bar{p}-p_{\text {ext }}=\beta \sqrt{A}-p^{*}, \quad \text { where }\left\{\begin{array}{l}
\beta=\frac{\sqrt{\pi} h E}{\left(1-\nu^{2}\right) A_{0}} \\
p^{*}=\beta \sqrt{A_{0}}
\end{array}\right.
$$

Henceforth, in view of (18), we have that

$$
\psi(A)=\beta \sqrt{A}-p^{*}
$$

The characteristic variables now are

$$
W_{1,2}=\bar{u} \pm 2 \sqrt{\frac{2}{\rho}}\left(\sqrt{\bar{p}-p_{e x t}+p^{*}}-\sqrt{p^{*}}\right) .
$$

In our simulations we have adopted this algebraic model. A presentation of other models that make use of differential laws linking $\bar{p}$ to $A$ and its time derivatives is reported in [4].

Remark 2.1 In the blood flow context, other algebraic relationships between $\bar{p}$ and $A$, possibly featuring a better accordance with experiments, can be found in [6], [9] and [19].

When considering a $2 \mathrm{D}$ geometry like the one shown in Fig. 2, equations (17) are still valid provided we now take $A=2\left(R_{0}+\eta_{r}\right)$. The algebraic relation (22) becomes

$$
\psi(A)=\beta A-p^{*} \quad \text { where }\left\{\begin{array}{l}
\beta=\frac{h E}{2\left(1-\nu^{2}\right) R_{0}^{2}} \\
p^{*}=\beta A_{0}
\end{array}\right.
$$

and the characteristic variables are $W_{1,2}=\bar{u} \pm 2 \sqrt{\frac{1}{\rho}}\left(\sqrt{\bar{p}-p_{\text {ext }}+p^{*}}-\sqrt{p^{*}}\right)$.

\subsection{Energy conservation for the 1D model}

Here we derive an a priori estimate for the solution of system (17) in the interval $I=[a, b]$ under the hypotheses that $\forall t>0, \quad \lambda_{1}>0, \lambda_{2}<0$ (subcritical flow regime) and the area $A$ maintains positive.

Remark 2.2 The assumption $\lambda_{1}>0$ and $\lambda_{2}<0$ is sound in hemodynamics since $c_{1}$ is much greater than $|\bar{u}|$ in physiologic conditions.

We will consider the following initial and boundary conditions

$$
\begin{array}{ll}
\text { initial conditions } & A(0, z)=A^{0}, \quad Q(0, z)=Q^{0} \quad a \leq z \leq b \\
\text { boundary conditions } & W_{1}=g_{1} \text { at } z=a \\
W_{2}=g_{2} \text { at } z=b
\end{array}
$$

We define the energy of the $1 \mathrm{D}$ model, for each $t>0$, as

$$
\mathcal{E}(t)=\frac{1}{2} \rho \int_{a}^{b} A(t, z) \bar{u}^{2}(t, z) d z+\int_{a}^{b} \Psi(A(t, z)) d z
$$

where

$$
\Psi(A)=\int_{A_{0}}^{A} \psi(\zeta) d \zeta
$$

Owing to (18) we may observe that

$$
\Psi\left(A_{0}\right)=\Psi^{\prime}\left(A_{0}\right)=0 \quad \text { and } \Psi^{\prime \prime}(A)>0 \quad \forall A>0 .
$$

Therefore $\Psi(A)$ is always positive and $\mathcal{E}(t)$ is a positive function for all $Q$ and $A>0$ at each $t>0$. The following Lemma holds 
Lemma 2.1 In the case $\alpha=1$, system (17), supplied with an algebraic pressure-area relationship like in (18), satisfies the following conservation property

$$
\mathcal{E}(T)+\rho K_{R} \int_{0}^{T} \int_{a}^{b} \bar{u}^{2} d z d t+\left.\int_{0}^{T} Q\left(\bar{p}-p_{e x t}+\frac{1}{2} \rho \bar{u}^{2}\right)\right|_{a} ^{b} d t=\mathcal{E}(0)
$$

Obviously, $\mathcal{E}(0)$ depends only on the initial data $A^{0}$ and $Q^{0}$.

\section{Proof.}

Let's multiply the second equation of (17) by $\bar{u}$ and integrate over $I$. We will analyse separately the four terms.

- First term

$$
\begin{aligned}
& I_{1}=\int_{a}^{b} \frac{\partial(A \bar{u})}{\partial t} \bar{u} d z=\frac{1}{2} \int_{a}^{b} A \frac{\partial \bar{u}^{2}}{\partial t} d z+\int_{a}^{b} \frac{\partial A}{\partial t} \bar{u}^{2} d z= \\
& \frac{1}{2} \frac{d}{d t} \int_{a}^{b} A \bar{u}^{2} d z+\frac{1}{2} \int_{a}^{b} \bar{u}^{2} \frac{\partial A}{\partial t} d z
\end{aligned}
$$

- Second term

$$
\begin{gathered}
I_{2}=\alpha \int_{a}^{b} \frac{\partial\left(A \bar{u}^{2}\right)}{\partial z} \bar{u} d z=\alpha\left[\int_{a}^{b} \frac{\partial(A \bar{u})}{\partial z} \bar{u}^{2} d z+\int_{a}^{b} A \bar{u}^{2} \frac{\partial \bar{u}}{\partial z} d z\right]= \\
\alpha\left[\frac{1}{2} \int_{a}^{b} \frac{\partial(A \bar{u})}{\partial z} \bar{u}^{2} d z+\frac{1}{2} \int_{a}^{b} \frac{\partial A}{\partial z} \bar{u}^{3} d z+\frac{3}{2} \int_{a}^{b} A \bar{u}^{2} \frac{\partial \bar{u}}{\partial z} d z\right]= \\
\alpha\left[\frac{1}{2} \int_{a}^{b} \frac{\partial Q}{\partial z} \bar{u}^{2} d z+\frac{1}{2} \int_{a}^{b} \frac{\partial\left(A \bar{u}^{3}\right)}{\partial z} d z\right]
\end{gathered}
$$

Now, using the first equation in (17) we obtain

$$
I_{2}=\frac{\alpha}{2}\left[-\int_{a}^{b} \frac{\partial A}{\partial t} \bar{u}^{2} d z+\left.\left(A \bar{u}^{3}\right)\right|_{a} ^{b}\right]
$$

- Third term. Using (18) and the fact that $p_{\text {ext }}$ is independent of $z$ we have

$$
\begin{aligned}
I_{3}=\int_{a}^{b} \frac{A}{\rho} \frac{\partial \bar{p}}{\partial z} \bar{u} d z=\frac{1}{\rho} \int_{a}^{b} A \frac{\partial}{\partial z}\left(\bar{p}-p_{\text {ext }}\right) \bar{u} d z & = \\
& \frac{1}{\rho}\left[-\int_{a}^{b} \frac{\partial Q}{\partial z} \psi(A) d z+\left.\left(\bar{p}-p_{\text {ext }}\right) Q\right|_{a} ^{b}\right]
\end{aligned}
$$

Again, using the first of (17) we have

$$
I_{3}=\frac{1}{\rho}\left[\int_{a}^{b} \frac{\partial A}{\partial t} \psi(A) d z+\left.\left(\bar{p}-p_{e x t}\right) Q\right|_{a} ^{b}\right]=\frac{1}{\rho}\left[\frac{d}{d t} \int_{a}^{b} \Psi(A) d z+\left.\left(\bar{p}-p_{\text {ext }}\right) Q\right|_{a} ^{b}\right]
$$

- Fourth term

$$
I_{4}=\int_{a}^{b} K_{R} \frac{Q}{A} \bar{u} d z=K_{R} \int_{a}^{b} \bar{u}^{2} d z
$$


In the case $\alpha=1$, by summing the four terms we obtain the following equality

$$
\frac{1}{2} \rho \frac{d}{d t} \int_{a}^{b} A \bar{u}^{2} d z+\frac{d}{d t} \int_{a}^{b} \Psi(A) d z+\rho K_{R} \int_{a}^{b} \bar{u}^{2} d z+\left.Q\left(\bar{p}-p_{\text {ext }}+\frac{1}{2} \rho \bar{u}^{2}\right)\right|_{a} ^{b}=0
$$

Integrating equation (35) in time between 0 and $T$ we obtain the desired result. $\diamond$

In order to draw an energy inequality from (28), we need to investigate the sign of the different terms. The first two of them are clearly positive. Concerning the third one let us analyse the homogeneous case (i.e. the case where $g_{1}=g_{2}=0$ ).

We will rewrite the boundary term in (28) as a function of $A, \psi(A)$ and $c_{1}$ (which, in its turn, depends on $A$ and $\psi(A)$, see (19)).

If $g_{1}=g_{2}=0$ in $(25)$, then

$$
\begin{array}{llll}
\text { at } \mathrm{z}=\mathrm{a} & W_{1}=\bar{u}+\int_{A_{0}}^{A} \frac{c_{1}(\zeta)}{\zeta} d \zeta=0 & \Longrightarrow & \bar{u}(t, a)=-\int_{A_{0}}^{A} \frac{c_{1}(\zeta)}{\zeta} d \zeta \\
\text { at } \mathrm{z}=\mathrm{b} & W_{2}=\bar{u}-\int_{A_{0}}^{A} \frac{c_{1}(\zeta)}{\zeta} d \zeta=0 & \Longrightarrow & \bar{u}(t, b)=\int_{A_{0}}^{A} \frac{c_{1}(\zeta)}{\zeta} d \zeta
\end{array}
$$

Then, using (18)

$$
\left.Q\left(\bar{p}-p_{e x t}+\frac{1}{2} \rho \bar{u}^{2}\right)\right|_{a} ^{b}=F(A(t, a))+F(A(t, b))
$$

where

$$
F(A)=A \int_{A_{0}}^{A} \frac{c_{1}(\zeta)}{\zeta} d \zeta\left[\psi(A)+\frac{1}{2} \rho\left(\int_{A_{0}}^{A} \frac{c_{1}(\zeta)}{\zeta} d \zeta\right)^{2}\right]
$$

We recall that we are assuming $\lambda_{1}>0$ and $\lambda_{2}<0$ (sub-critical flow regime). Then, from (19), we have $|\bar{u}|<c_{1}$ which implies at both $z=a$ and $z=b$

$$
\left|\int_{A_{0}}^{A} \frac{c_{1}(\zeta)}{\zeta} d \zeta\right|<c_{1}(A)
$$

We are now in the position to conclude with the following result

Lemma 2.2 If the function $\psi(A)$ is chosen in such a way that $F(A)>0$, for all $A>0$ which satisfy (38), then the energy inequality

$$
\mathcal{E}(T)+\rho K_{R} \int_{0}^{T} \int_{a}^{b} \bar{u}^{2} d z d t \leq \mathcal{E}(0)
$$

holds for system (17).

The pressure-area relationship given in (22) satisfies the hypotheses of Lemma 2.2. In fact, in this case

$$
\Psi(A)=\beta\left(\sqrt{A}-\sqrt{A_{0}}\right), \quad c_{1}^{2}=\frac{\beta}{2 \rho} \sqrt{A}
$$

and

$$
\int_{A_{0}}^{A} \frac{c_{1}(\zeta)}{\zeta} d \zeta=\int_{A_{0}}^{A} \sqrt{\frac{\beta}{2 \rho}} \zeta^{-\frac{3}{4}} d \zeta=4 \sqrt{\frac{\beta}{2 \rho}}\left(A^{\frac{1}{4}}-A_{0}^{\frac{1}{4}}\right) .
$$


Condition (38) becomes

$$
\left|4 \sqrt{\frac{\beta}{2 \rho}}\left(A^{\frac{1}{4}}-A_{0}^{\frac{1}{4}}\right)\right|<\sqrt{\frac{\beta}{2 \rho}} A^{\frac{1}{4}} \quad \text { and then } \quad \frac{4}{5} A_{0}^{\frac{1}{4}}<A^{\frac{1}{4}}<\frac{4}{3} A_{0}^{\frac{1}{4}}
$$

On the other hand we have

$$
\begin{gathered}
F(A)=A 4 \sqrt{\frac{\beta}{2 \rho}}\left(A^{\frac{1}{4}}-A_{0}^{\frac{1}{4}}\right)\left[\beta\left(A^{\frac{1}{2}}-A_{0}^{\frac{1}{2}}\right)+\frac{\rho}{2} 16 \frac{\beta}{2 \rho}\left(A^{\frac{1}{4}}-A_{0}^{\frac{1}{4}}\right)^{2}\right]= \\
4 \beta \sqrt{\frac{\beta}{2 \rho}} A\left(A^{\frac{1}{4}}-A_{0}^{\frac{1}{4}}\right)\left[\left(A^{\frac{1}{4}}-A_{0}^{\frac{1}{4}}\right)\left(A^{\frac{1}{4}}+A_{0}^{\frac{1}{4}}\right)+4\left(A^{\frac{1}{4}}-A_{0}^{\frac{1}{4}}\right)^{2}\right]= \\
4 \beta \sqrt{\frac{\beta}{2 \rho}} A\left(A^{\frac{1}{4}}-A_{0}^{\frac{1}{4}}\right)^{2}\left(5 A^{\frac{1}{4}}-3 A_{0}^{\frac{1}{4}}\right)
\end{gathered}
$$

Then, condition $F(A)>0$ gives

$$
A^{\frac{1}{4}}>\frac{3}{5} A_{0}^{\frac{1}{4}}
$$

whose satisfaction is a consequence of (40). Therefore, in that case the 1D model is stable.

More generally, under the same relation (22), we can prove an energy estimate also in the case of non homogeneous boundary conditions. We have the following result

Lemma 2.3 If the pressure-area relationship is given by (22), and the boundary data satisfy

$$
g_{1}(t)>-4 \sqrt{\frac{p^{*}}{2 \rho}} \quad \text { and } \quad g_{2}(t)<4 \sqrt{\frac{p^{*}}{2 \rho}} \quad \forall t>0
$$

then there exists a positive function $G=G\left(g_{1}, g_{2}, p^{*}\right)$ such that

$$
\mathcal{E}(T)+\rho K_{R} \int_{0}^{T} \int_{a}^{b} \bar{u}^{2} d z d t \leq \mathcal{E}(0)+\int_{0}^{T} G\left(g_{1}(t), g_{2}(t), p^{*}\right) d t .
$$

\section{Proof.}

We will consider only the case where $g_{1} \neq 0$ and $g_{2}=0$, since the most general case may be derived in a similar fashion. We recall that relationship (22) satisfies the hypotheses on $F(A)$ of Lemma 2.2. Then from (35) we obtain the following inequality

$$
\begin{gathered}
\frac{\rho}{2} \frac{d}{d t} \int_{a}^{b} A \bar{u}^{2} d z+\frac{d}{d t} \int_{a}^{b} \Psi(A) d z+\rho K_{R} \int_{a}^{b} \bar{u}^{2} d z \leq \\
\left.Q\left(\bar{p}-p_{\text {ext }}+\frac{1}{2} \rho \bar{u}^{2}\right)\right|_{z=a} \leq\left.\left(A|\bar{u}||\psi(A)|+\frac{1}{2} \rho A|\bar{u}|^{3}\right)\right|_{z=a}
\end{gathered}
$$

We have then at $z=a$ (from (23) and (25))

$$
\bar{u}+2 \sqrt{\frac{2}{\rho}}\left(\sqrt{\bar{p}-p_{e x t}+p^{*}}-\sqrt{p^{*}}\right)=g_{1}
$$

thus, from (18)

$$
\bar{u}=g_{1}-2 \sqrt{\frac{2}{\rho}}\left(\sqrt{\psi(A)+p^{*}}-\sqrt{p^{*}}\right) .
$$


On the other hand, $\lambda_{1}=\bar{u}+\frac{1}{\sqrt{2 \rho}} \sqrt{\bar{p}-p_{e x t}+p^{*}}>0$, and then

$$
\bar{u}>-\frac{1}{\sqrt{2 \rho}} \sqrt{\psi(A)+p^{*}}
$$

Combining (44) and (45) we have

$$
-\frac{1}{\sqrt{2 \rho}} \sqrt{\psi(A)+p^{*}}<g_{1}-2 \sqrt{\frac{2}{\rho}}\left(\sqrt{\psi(A)+p^{*}}-\sqrt{p^{*}}\right)
$$

and then

$$
\sqrt{\psi(A)+p^{*}}<f_{1}\left(g_{1}, p^{*}\right)
$$

where

$$
f_{1}\left(g_{1}, p^{*}\right)=\frac{\sqrt{2 \rho}}{3} g_{1}+\frac{4}{3} \sqrt{p^{*}} .
$$

The assumptions (41) are necessary conditions for the eigenvalues of system (17) being of opposite sign.

Furthermore, we deduce from $(22)$ that $\psi(A)>-p^{*}$. The following inequalities thus hold at $z=a$

$$
\begin{aligned}
& |\psi(A)| \leq \max \left\{p^{*},\left|f_{1}^{2}\left(g_{1}, p^{*}\right)-p^{*}\right|\right\} \\
& |\bar{u}|=\left|g_{1}-2 \sqrt{\frac{2}{\rho}}\left(\sqrt{\psi(A)+p^{*}}-\sqrt{p^{*}}\right)\right| \leq\left|g_{1}\right|+2 \sqrt{\frac{2}{\rho}}\left(\sqrt{p^{*}}+f_{1}\left(g_{1}, p^{*}\right)\right) \\
& A=\frac{1}{\beta^{2}}\left(\psi(A)+p^{*}\right)^{2} \leq \frac{1}{\beta^{2}} f_{1}^{4}\left(g_{1}, p^{*}\right)
\end{aligned}
$$

and the right hand side in (43) may thus be expressed by a positive function of the boundary data $g_{1}$ and the reference pressure at rest $p^{*}=\beta \sqrt{A_{0}}$, which we have indicate by $G\left(g_{1}, p^{*}\right)$. We obtain then the desired stability inequality. $\diamond$

\subsection{Entropy function for the 1D model}

If we take again the differential problem (17) and we carry out derivations similar to those illustrated in (29)-(35), considering now the integrals over an arbitrary interval $[z, z+d z] \subset[a, b]$, we may derive a relation analogous to (35). Then, passing to the limit as $d z \rightarrow 0$, the following pointwise differential relation may be deduced

$$
\frac{\partial}{\partial t}\left(\frac{1}{2} \rho A \bar{u}^{2}+\Psi(A)\right)+\frac{\partial}{\partial z}\left[Q\left(\psi(A)+\frac{1}{2} \rho \bar{u}^{2}\right)\right]=-\rho K_{R} \bar{u}^{2}
$$

which can be interpreted as an entropy balance equation for the hyperbolic system. Indeed, the function

$$
s(A, Q)=\frac{1}{2} \rho A \bar{u}^{2}+\Psi(A)
$$

in an entropy for system (17), with an associated flux

$$
F_{s}(A, Q)=Q\left(\psi(A)+\frac{1}{2} \rho \bar{u}^{2}\right)
$$

The term $\rho K_{R} \bar{u}^{2}$ may be recognised as a dissipative term. 


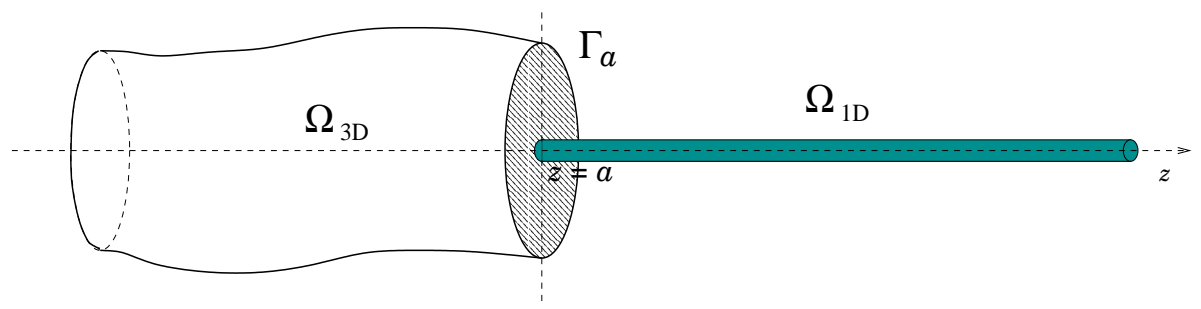

Figure 3: Coupling a 3D model with a 1D model

\section{Coupling the 3D model with the $1 \mathrm{D}$ model}

We consider now two domains $\Omega_{3 D}$ and $\Omega_{1 D}$ as in Fig. 3 and we solve, in the first, the 3D fluid-structure model while in the second we consider the simplified 1D model.

On the right side of $\Gamma_{a}$ the 1D model supplies the quantities $A\left(a^{+}\right), \quad Q\left(a^{+}\right), \quad \bar{p}\left(a^{+}\right)=\psi(A)$ and $\bar{u}\left(a^{+}\right)=Q / A$. We define, then, the same quantities also on the left side of $\Gamma_{a}$ as

$$
\begin{aligned}
A\left(a^{-}\right)=\left|\Gamma_{a}\right| ; & \bar{u}\left(a^{-}\right) & =\frac{1}{\left|\Gamma_{a}\right|} \int_{\Gamma_{a}} \mathbf{u} \cdot \mathbf{n} d \sigma \\
\bar{p}\left(a^{-}\right)=\frac{1}{\left|\Gamma_{a}\right|} \int_{\Gamma_{a}} p d \sigma ; & Q\left(a^{-}\right) & =\left|\Gamma_{a}\right| \bar{u}\left(a^{-}\right) .
\end{aligned}
$$

Moreover, we assume that at $\Gamma_{a}$ the wall displacement is a function of the section area,

$$
\left.\boldsymbol{\eta}\right|_{\Gamma_{a}}=\mathbf{g}\left(A\left(a^{-}\right)\right),
$$

being $\mathbf{g}$ a given function. For instance, we can assume that $\Gamma_{a}$ is a circle in which case $\left.\boldsymbol{\eta}\right|_{\Gamma_{a}}=$ $\left(\sqrt{A\left(a^{-}\right) / \pi}-R_{0}\right) \mathbf{e}_{r}$.

It is a priori reasonable to look for the continuity of the following quantities at the interface $\Gamma_{a}$ :
[A] area :
$A\left(a^{-}\right)=A\left(a^{+}\right)$
$[\mathrm{B}]$ mean pressure :
$\bar{p}\left(a^{-}\right)=\bar{p}\left(a^{+}\right)$
[C] flux :
$Q\left(a^{-}\right)=Q\left(a^{+}\right)$
$[D]$ entering characteristic :
$\bar{u}\left(a^{-}\right)+2 \sqrt{\frac{2}{\rho}}\left(\sqrt{\bar{p}\left(a^{-}\right)-p_{e x t}+p^{*}}-\sqrt{p^{*}}\right)=W_{1}\left(a^{+}\right)$

Since in the 1D model viscous terms have been (partially) neglected, the variable $\bar{p}$ can be either interpreted as a mean pressure or as a mean normal stress. The condition $[\mathrm{B}]$ may then be replaced with the continuity of the averaged normal stress, i.e. $\bar{\sigma}=\overline{\mathbf{T} \cdot \mathbf{n} \cdot \mathbf{n}}$. Analogously, the characteristic variable on the left hand side can be calculated using the averaged normal stress in place of the mean pressure. We have then two conditions alternative to B and D, respectively. Namely,

[B1] averaged normal stress : $\quad \bar{\sigma}\left(a^{-}\right)=\bar{p}\left(a^{+}\right)$

[D1] char. entering variable : $\quad \bar{u}\left(a^{-}\right)+2 \sqrt{\frac{2}{\rho}}\left(\sqrt{\left.\bar{\sigma}\left(a^{-}\right)-p_{\text {ext }}+p^{*}\right)}-\sqrt{p^{*}}\right)=W_{1}\left(a^{+}\right)$

In view of the splitting procedure that will be described in Section 3.1 to solve the coupled 3D-1D model, we are allowed to enforce, at the interface point $a$, only those conditions that will generate well posed individual subproblems in $\Omega_{3 D}$ and $\Omega_{1 D}$.

To this aim, we advocate four different set of coupling conditions:

- Interaction Model 1: conditions A, B, D

We note that $\mathrm{B}$ and $\mathrm{D}$ imply the continuity of $\bar{u}$. With the further continuity of A we obtain that of $Q$. Thus also $\mathrm{C}$ is satisfied. 
- Interaction Model 2: conditions A, C, D

We note that $\mathrm{A}$ and $\mathrm{C}$ imply the continuity of $\bar{u}$. If we further add $\mathrm{D}$ we have the continuity of $\bar{p}$. Thus also B is satisfied.

- Interaction Model 3: conditions A, B1, D1

We note that B1 and D1 imply the continuity of $\bar{u}$ and, with the continuity of A we obtain that of $Q$. Thus also $\mathrm{C}$ is satisfied.

- Interaction Model 4: conditions A, C, D1

We note that $\mathrm{A}$ and $\mathrm{C}$ imply the continuity of $\bar{u}$. If we further add $\mathrm{D}$ we have the continuity of $\bar{\sigma}$. Thus also B1 is satisfied.

\subsection{Sub-domain iterations between 1D and 3D models}

Each Interaction Model presented above can be split into two subproblems:

- A 1D problem with condition D (or D1) as boundary condition at the interface and an absorbing condition on the right end (i.e. zero entering characteristic variable). With this choice, the hyperbolic system (17) is well posed, as we have shown in Lemma 2.3.

- A 3D fluid-structure problem with condition A as boundary condition for the structure equation and condition B (B1) or C, depending on which interaction model we consider, as boundary condition for the Navier-Stokes equations. On the inflow section $\mathcal{S}_{1}$ we might impose ordinary Dirichlet or natural conditions; for instance we could assign the velocity profile for the fluid and the displacement of the wall.

With these choices the structure problem turns out to be well posed, while conditions B or $\mathrm{C}$ as such are not sufficient to close the fluid equations since they only provide average and not pointwise values on $\Gamma_{a}$.

A possible way to impose such defective boundary conditions to the fluid equations, known as "do nothing" approach, is proposed in [7] and analysed in the context of blood flow in [13]. We have adopted this approach to impose the condition [B1] in the simulations presented in section 5 .

An alternative approach based on Lagrange multipliers is investigated in [3].

We can then iterate between the two sub-domains to get the global solution at each time step. For instance, referring to the Interaction Model 3 (which is the one we have implemented) the iterative algorithm reads as follows: given the solution of the coupled problem (let's say $\mathbf{u}^{n}, p^{n}, \boldsymbol{\eta}^{n}$ for the 3D model and $Q^{n}, A^{n}$ for the $1 \mathrm{D}$ one) and setting $\mathbf{u}_{0}^{n+1}=\mathbf{u}^{n}, p_{0}^{n+1}=p^{n}$, and $\boldsymbol{\eta}_{0}^{n+1}=\boldsymbol{\eta}^{n}$

1. solve the 1D model (17) with condition D1 at the interface, evaluating $W_{1}\left(a^{+}\right)$as a function of $\mathbf{u}_{0}^{n+1}, p_{0}^{n+1}, \boldsymbol{\eta}_{0}^{n+1}$; we then obtain a solution $Q_{1}^{n+1}$ and $A_{1}^{n+1}$;

2. solve the $3 \mathrm{D}$ problem with boundary conditions $\mathrm{A}$ and $\mathrm{B} 1$ at the interface, evaluating $A\left(a^{-}\right)$ and $\bar{\sigma}\left(a^{-}\right)$as functions of $A_{1}^{n+1}$ and $Q_{1}^{n+1}$. We obtain a solution $\mathbf{u}_{1}^{n+1}, p_{1}^{n+1}, \boldsymbol{\eta}_{1}^{n+1}$.

We iterate until the coupling conditions are satisfied within a fixed tolerance. We can eventually add a relaxation step on the variable $W_{1}\left(a^{+}\right)$.

\section{Finite elements discretisation of the $3 \mathrm{D}$ and $1 \mathrm{D}$ models}

In this section we will present the schemes used for the numerical solution of the coupled problem together with some numerical results on the interaction between the $1 \mathrm{D}$ reduced model and the full fluid-structure coupled model for both the 2D and the 3D case. 
The numerical solution of the 1D model has been obtained by a standard Lax-Wendroff scheme ([5]). Writing the system (17) in conservative form:

$$
\frac{\partial \mathbf{U}}{\partial t}+\frac{\partial}{\partial z} \mathbf{F}(\mathbf{U})=\mathbf{B}(\mathbf{U})
$$

where

$$
\mathbf{U}=\left[\begin{array}{l}
A \\
Q
\end{array}\right], \quad \mathbf{F}(\mathbf{U})=\left[\begin{array}{c}
Q \\
\alpha \frac{Q^{2}}{A}+\int_{A_{0}}^{A} c_{1}^{2}(y) d y
\end{array}\right], \quad \mathbf{B}(\mathbf{U})=\left[\begin{array}{c}
0 \\
-K_{R} \frac{Q}{A}
\end{array}\right],
$$

the Lax-Wendroff scheme may be written as

$$
\begin{aligned}
\mathbf{U}^{n+1}=\mathbf{U}^{n}+\Delta t\left(-\frac{\partial \mathbf{F}}{\partial z}+\mathbf{B}\right)^{n}+\frac{\Delta t^{2}}{2}\left[\frac{\partial}{\partial z}\left(-\left(\frac{\partial \mathbf{F}}{\partial \mathbf{U}}\right)^{2} \frac{\partial \mathbf{U}}{\partial z}-\frac{\partial \mathbf{F}}{\partial \mathbf{U}} \mathbf{B}\right)+\right. & \left.\frac{\partial \mathbf{B}}{\partial \mathbf{U}}\left(-\frac{\partial \mathbf{F}}{\partial \mathbf{U}} \frac{\partial \mathbf{U}}{\partial z}+\mathbf{B}\right)\right]^{n} .
\end{aligned}
$$

where $\mathbf{U}^{n}$ denotes the numerical solution at time step $t=t^{n}$.

We have used standard linear finite elements for the space discretisation. Even if the temporal discretisation is explicit, we need to invert a tridiagonal mass matrix. A characteristic boundary treatment has been adopted and an extrapolation of the outgoing characteristic variable has been used to solve the compatibility condition.

For the 2D simulations of the fluid-structure interaction problem (1), (2) and (3), we have considered a conforming finite element discretisation. In particular, for the structure equation (3) we have used $\mathbb{P}^{1}$ finite elements in space and a Newmark scheme in time, that is

$$
\begin{aligned}
& \eta^{n+1}=\eta^{n}+\Delta t \dot{\eta}^{n}+\Delta t^{2}\left[\beta_{N} f^{n+1}+\left(\frac{1}{2}-\beta_{N}\right) f^{n}\right] \\
& \dot{\eta}^{n+1}=\dot{\eta}^{n}+\Delta t\left[\gamma_{N} f^{n+1}+\left(1-\gamma_{N}\right) f^{n}\right]
\end{aligned}
$$

being $\left(\eta^{n}, \dot{\eta}^{n}\right)$ an approximation of $\left(\eta_{r}\left(t^{n}\right), \dot{\eta}_{r}\left(t^{n}\right)\right)$ and

$$
f^{n}=\frac{k G h}{\rho_{w} h} \frac{\partial^{2} \eta^{n}}{\partial z^{2}}-\frac{E}{\rho_{w}\left(1-\nu^{2}\right) R_{0}^{2}} \eta^{n}+\gamma \frac{\partial^{2} \dot{\eta}^{n}}{\partial z^{2}}+\hat{\Phi}^{n}
$$

where $\hat{\Phi}^{n}$ is the forcing term at time $t^{n}$. The parameters $\beta_{N}$ and $\gamma_{N}$ of the scheme have been taken equal to $\beta_{N}=1 / 4$ and $\gamma_{N}=1 / 2$. We have shown in [11] that, with this choice, the Newmark scheme applied to equation (3) is unconditionally stable (at least in the case of homogeneous Dirichlet boundary conditions).

For the fluid we have adopted an "Arbitrary Lagrangian Eulerian" description and we have considered a Finite Element approximation with $\mathbb{P}^{1} i s o \mathbb{P}^{2}$ elements for the velocity field and $\mathbb{P}^{1}$ elements for the pressure. The non-linear term is treated in a semi-implicit way and the equations are solved through the Yosida projection scheme described in [20].

We have considered the following implicit algorithm to couple the two equations.

Given the solution $\mathbf{u}^{n}, p^{n}, \eta^{n}, \dot{\eta}^{n}$, at time $t^{n}$ :

1. we extrapolate the displacement $\eta$ and the velocity $\dot{\eta}$ of the structure

$$
\eta_{0}^{n+1}=\eta^{n}+\Delta t \dot{\eta}^{n}, \quad \dot{\eta}_{0}^{n+1}=\dot{\eta}^{n}
$$

2. we update the mesh by solving a Laplace problem which provide the displacement $\mathbf{s}\left(\mathbf{x}^{\mathbf{n}}\right)$ of each point $\mathbf{x}^{\mathbf{n}} \in \Omega^{n}$ as follows

$$
\begin{cases}\Delta \mathbf{s}=0 & \text { in } \Omega \\ \mathbf{s}=\eta^{n+1}-\eta^{n} & \text { on } \Gamma_{w}\end{cases}
$$

and setting $\mathbf{x}^{n+1}=\mathbf{x}^{n}+\mathbf{s}\left(\mathbf{x}^{n}\right)$. 
3. we assign the velocity of the fluid on the interface $\Gamma_{w}$

$$
\mathbf{u}_{0}^{n+1}=\dot{\eta}_{0}^{n+1} \mathbf{e}_{r}
$$

4. we solve the fluid equations and we evaluate the forcing term $\hat{\Phi}_{0}^{n+1}$ of equation (3)

5. we solve the structural equation obtaining a better estimate $\tilde{\eta}_{1}^{n+1}$ and $\tilde{\eta}_{1}^{n+1}$ of the displacement and the velocity of the arterial wall, which allows to set

$$
\begin{aligned}
& \eta_{1}^{n+1}=\omega \tilde{\eta}^{n+1}+(1-\omega) \eta_{0}^{n+1} \\
& \dot{\eta}_{1}^{n+1}=\omega \tilde{\dot{\eta}}^{n+1}+(1-\omega) \dot{\eta}_{0}^{n+1}
\end{aligned}
$$

where $\omega$ is a relaxation parameter.

6. we go back to 2 and we iterate until the convergence of $\eta_{k}^{n+1}, \dot{\eta}_{k}^{n+1}, \mathbf{u}_{k}^{n+1}, p_{k}^{n+1}$.

The algorithm presented converge if $\omega$ is taken suitably small. We have observed that the highest value of $\omega$ under which we have convergence depends on the wall mass $\rho_{w}$ and the length $L$ of the tube but seems independent on the elastic constants of the structural model and on $\Delta t$. Table 1 shows the critical value of $\omega$ with respect to $\rho_{w}$ and $L$.

Table 1: Values of $\omega$ which guarantee convergence of the algorithm.

\begin{tabular}{|c|c|c|c|}
\hline & $L=2$ & $L=6$ & $L=10$ \\
\hline$\rho_{w}=50$ & $\omega \leq 1$ & $\omega \leq 1$ & $\omega \leq 1$ \\
\hline$\rho_{w}=10$ & $\omega \leq 1$ & $\omega \leq 1$ & $\omega \leq 0,9$ \\
\hline$\rho_{w}=5$ & $\omega \leq 1$ & $\omega \leq 1$ & $\omega \leq 0,6$ \\
\hline$\rho_{w}=1$ & $\omega \leq 1$ & $\omega \leq 0,4$ & $\omega \leq 0,1$ \\
\hline$\rho_{w}=0.5$ & $\omega \leq 0,9$ & $\omega \leq 0,2$ & $\omega \leq 0,09$ \\
\hline$\rho_{w}=0.1$ & $\omega \leq 0,4$ & $\omega \leq 0,05$ & $\omega \leq 0,01$ \\
\hline
\end{tabular}

For the 3D simulations, we have used the code SPECTRUM. As for the 2D model, the moving fluid domain is handled with an ALE formulation. At each time step, the code solves iteratively the coupled problem by treating simultaneously the fluid and the structure equations in a first step ("stagger") (enforcing the adherence of the fluid to the structure with a Lagrange multiplier technique), and in a second step it accounts for the deformation of the grid.

Concerning the coupling with the reduced 1D model, we have considered Model 3 described in section 3. We have made just one sub-iteration between 1D and 3D (2D) model at each time step.

We have noticed that a "brute force" imposition of the continuity of the area $A$ causes numerical instability, probably because of the discrepancy in the structural laws used in the two models. The matter is still a subject of investigation. For the computations we are going to illustrate, we have relaxed the constraint on the continuity of $A$ by adopting, in the $2 \mathrm{D}$ case, the absorbing boundary conditions (14)-(15) and, in the 3D case, natural boundary conditions on the transversal displacement.

\section{$5 \quad$ Numerical results and model assessment}

We will present two numerical tests.

Test 1: Pressure pulse in a compliant vessel 
In the $2 \mathrm{D}$ case, we have considered a rectangular domain of height $1 \mathrm{~cm}$ and length $L=6 \mathrm{~cm}$. The fluid is initially at rest and an over pressure of $15 \mathrm{mmHg}\left(2 \cdot 10^{4}\right.$ dynes $\left./ \mathrm{cm}^{2}\right)$ has been imposed at the inlet for 0.005 seconds. The viscosity of the fluid is equal to 0.035 poise, its density is $1 \mathrm{~g} / \mathrm{cm}^{3}$, the Young modulus of the structure is equal to $0.75 \cdot 10^{6} \mathrm{dynes} / \mathrm{cm}^{2}$, its Poisson coefficient is 0.5 , its density is $1.1 \mathrm{~g} / \mathrm{cm}^{3}$ and its thickness is $0.1 \mathrm{~cm}$.

In the $3 \mathrm{D}$ case, our computation has been made on a cylindrical domain of radius $R_{0}=0.5 \mathrm{~cm}$ and length $L=5 \mathrm{~cm}$, with the following physical parameters: fluid viscosity: 0.03 poise, fluid density: $1 \mathrm{~g} / \mathrm{cm}^{3}$, Young modulus of the structure: $3 \cdot 10^{6}$ dynes $/ \mathrm{cm}^{2}$, Poisson coefficient: 0.3 and structure density: $1.2 \mathrm{~g} / \mathrm{cm}^{3}$. Again, an over-pressure of $10 \mathrm{mmHg}\left(1.3332 \cdot 10^{4}\right.$ dynes $\left./ \mathrm{cm}^{2}\right)$ is imposed at the inlet for 0.005 seconds.

We have first simulated the fluid-structure interaction model without any coupling with the 1D reduced model. In this case, natural boundary conditions for the fluid (i.e. null normal stress) have been imposed on the outlet. Figures 4 and 5 show the fluid pressure and the domain deformation in the $3 \mathrm{D}$ and the $2 \mathrm{D}$ case respectively. For the sake of clarity, the displacements shown in Figure 4 are magnified by a factor 10 .

A pressure wave is generated (together with a deformation wave at the same velocity), which is reflected at the outflow section. Such a reflection is clearly a numerical side-effect due to the boundary conditions imposed at the outflow.

In Figures 6 and 7 we have considered the coupling with the 1D reduced model at the outflow section. Here the reflections are greatly reduced because the pressure wave is quite well absorbed by the $1 \mathrm{D}$ model.

Test 2: Pressure step in a compliant vessel - error analysis

We have considered a rectangular domain $\Omega=[0,12] \times[0,1]$, divided into two sub-rectangles $\Omega_{1}=[0,6] \times[0,1]$ and $\Omega_{2}=[6,12] \times[0,1]$. We have simulated the $2 \mathrm{D}$ fluid structure interaction problem in $\Omega$ when a pressure step of 5000 dynes $/ \mathrm{cm}^{2}, \forall t>0$, is imposed at the inlet on a fluid initially at rest. Let $(\mathbf{u}, p)$ be the numerical solution obtained for the fluid. We have then considered the fluid-structure problem in $\Omega_{1}$, coupled with the $1 \mathrm{D}$ reduced model at the outlet, with the same conditions at the inlet. The numerical solution obtained in this case is indicated as $\left(\mathbf{u}_{1}, p_{1}\right)$. We have compared then the two solutions on domain $\Omega_{1}$. Figure 8 shows the relative error in the $L^{2}$ norm between the two solutions, i.e.

$$
\varepsilon_{p}=\frac{\left\|p-p_{1}\right\|_{L^{2}\left(\Omega_{1}\right)}}{\|p\|_{L^{2}\left(\Omega_{1}\right)}}, \quad \varepsilon_{u}=\frac{\left\|\mathbf{u}-\mathbf{u}_{1}\right\|_{L^{2}\left(\Omega_{1}\right)}}{\|\mathbf{u}\|_{L^{2}\left(\Omega_{1}\right)}} .
$$

Both errors are less then 2.5\%. Their peaks correspond to the instant at which the pressure wave reaches the outflow section of $\Omega_{1}$. 

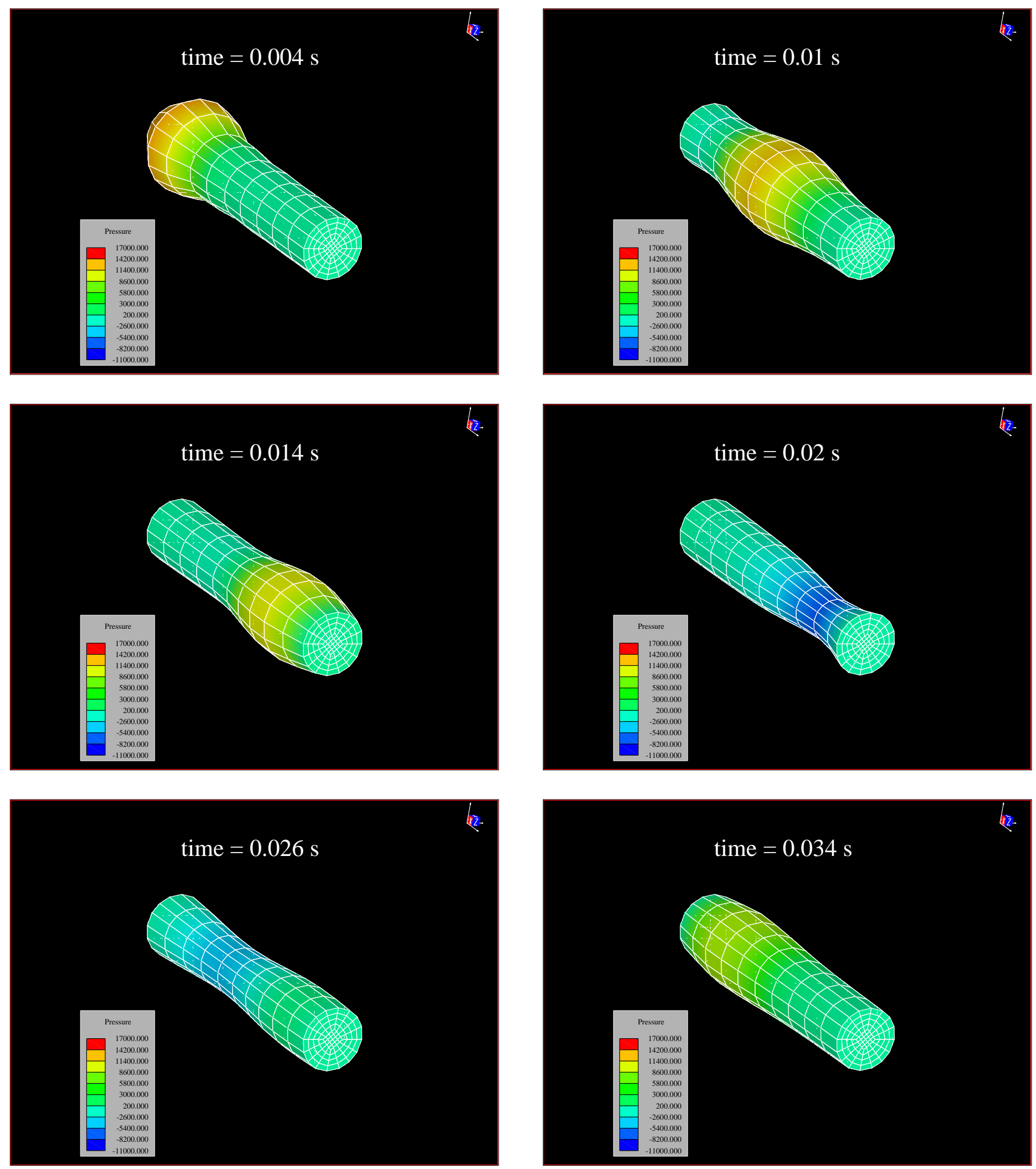

Figure 4: pressure pulse entering at the inflow and homogeneous Neumann conditions at the outflow: 3D simulation with SPECTRUM. The displacement of the structure has been magnified by a factor 10 


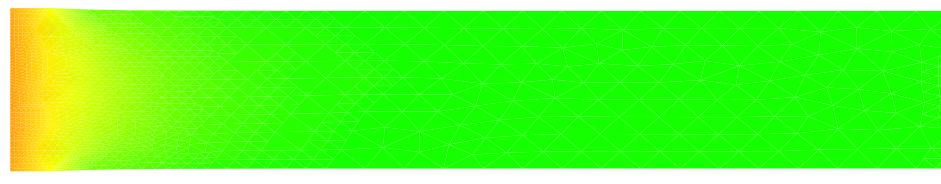

$$
t=1 \mathrm{~ms}
$$

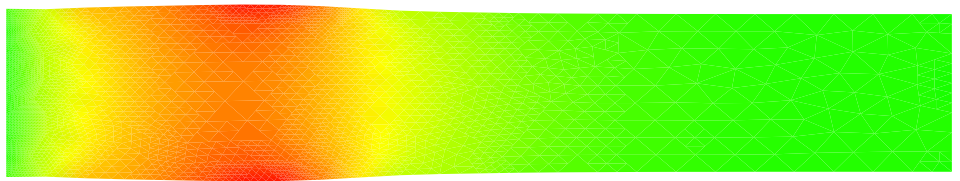

$$
t=6 \mathrm{~ms}
$$

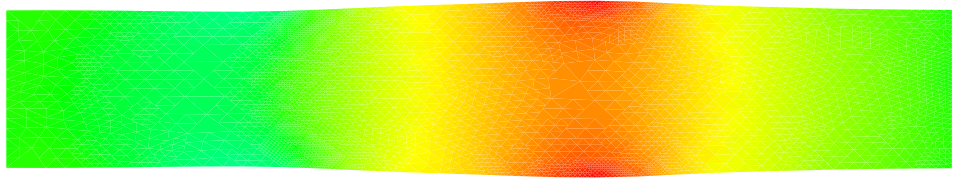

$$
t=11 \mathrm{~ms}
$$

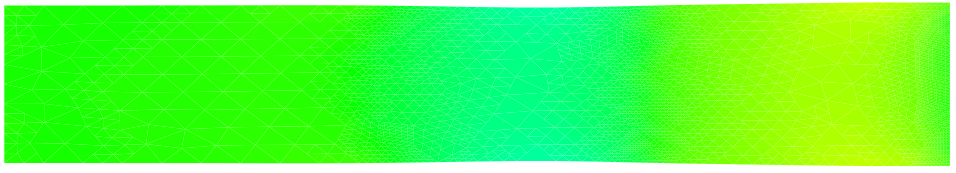

$t=16 \mathrm{~ms}$

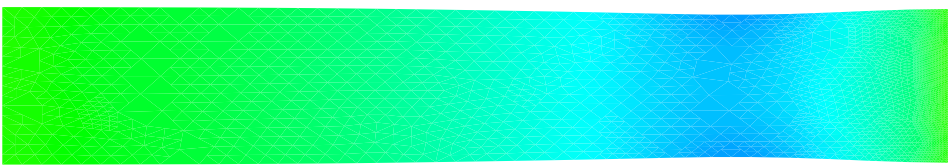

$t=21 \mathrm{~ms}$

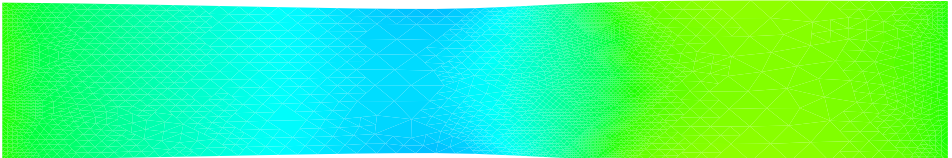

$t=26 m s$

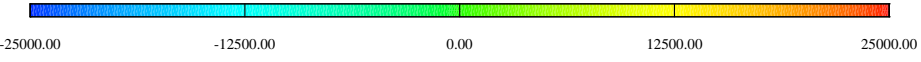

Figure 5: pressure pulse entering at the inflow and homogeneous Neumann conditions at the outflow; 2D simulation. Solutions every $5 \mathrm{~ms}$. 

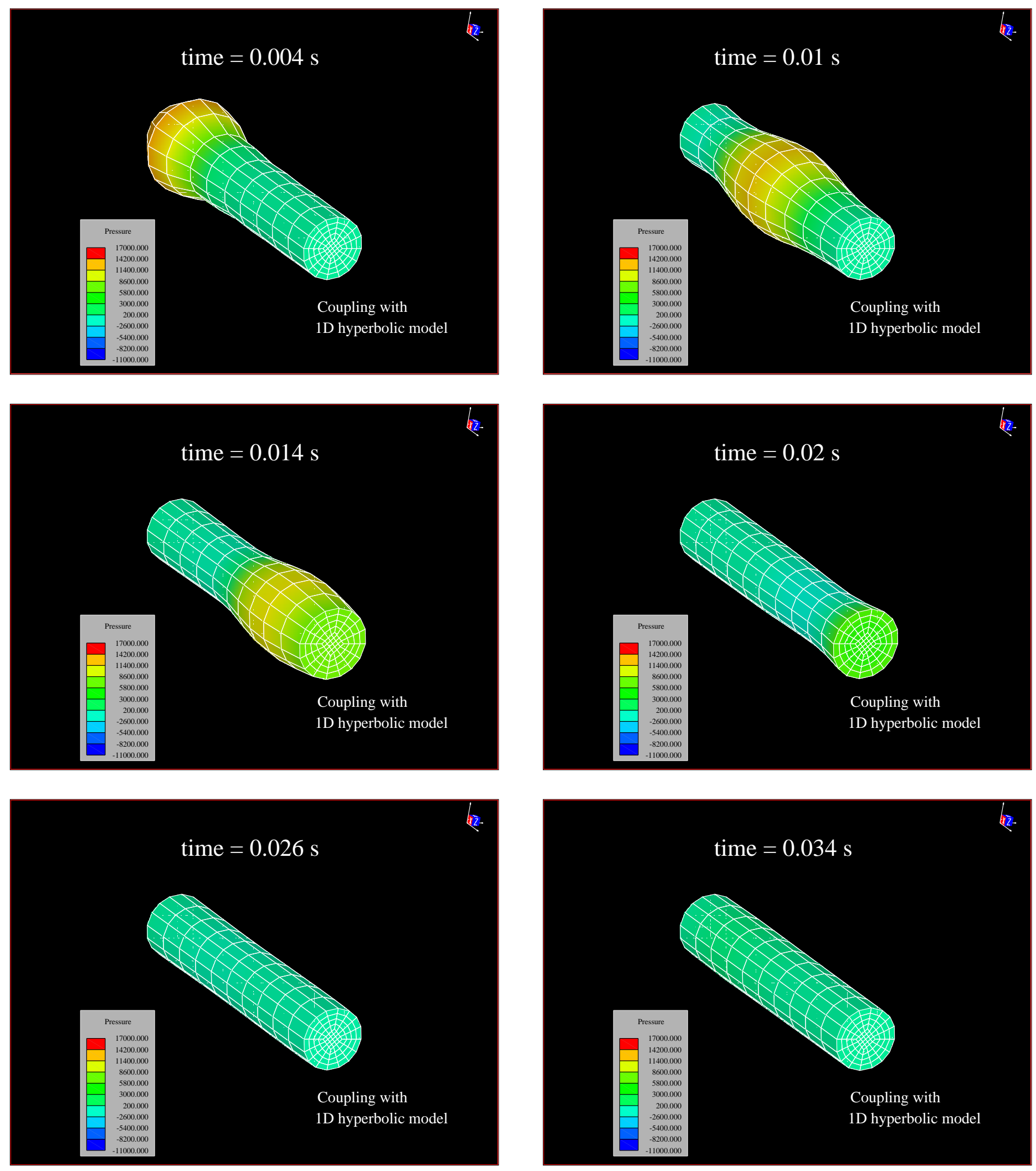

Figure 6: coupling 3D SPECTRUM simulation with the 1D reduced model 

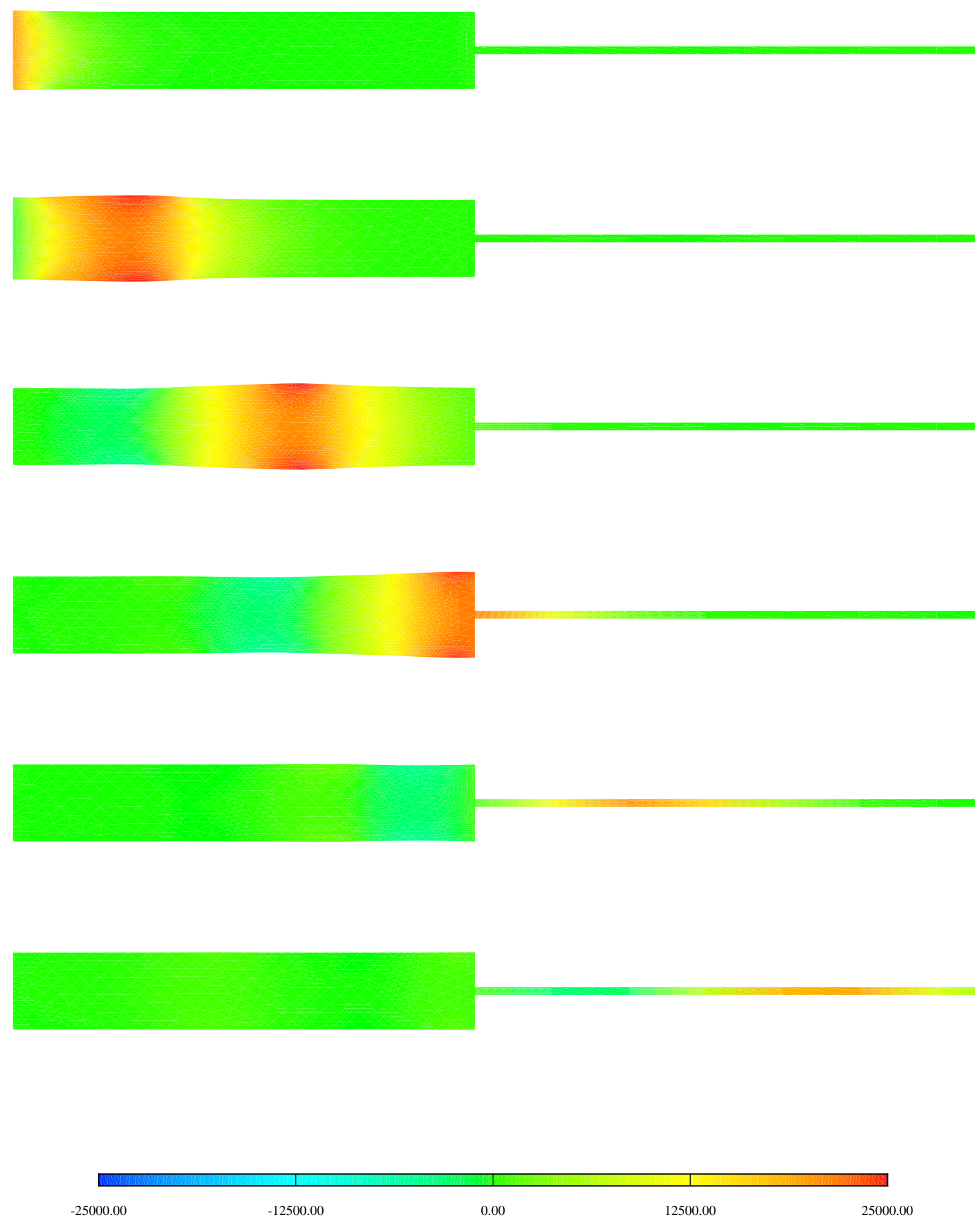

Figure 7: coupling 2D simulation with the 1D reduced model; solution every $5 \mathrm{~ms}$ 


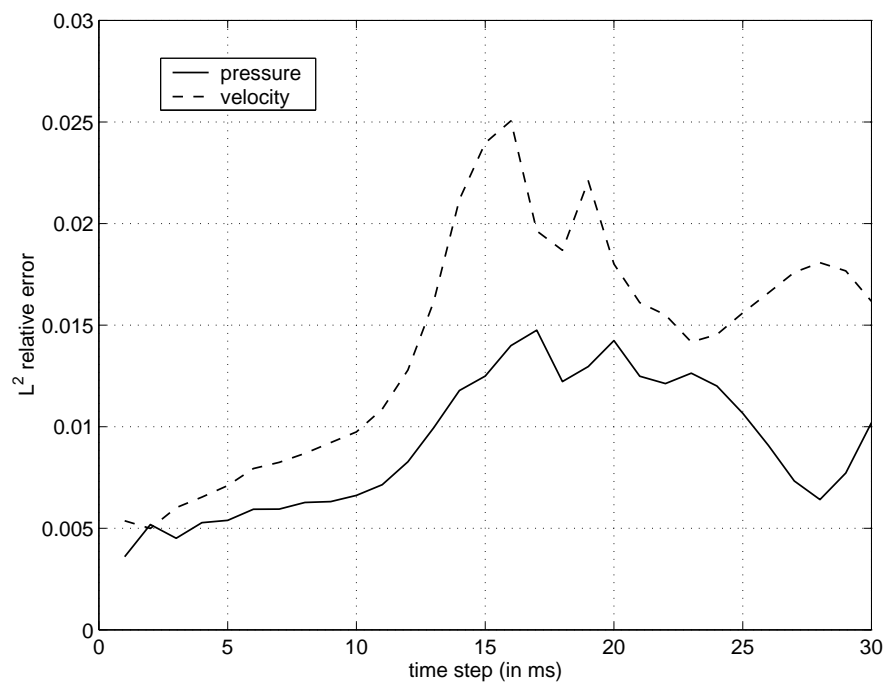

Figure 8: relative error in $L^{2}$ norm both for pressure and velocity field, between the solution of the coupled 2D/1D model and the solution of the fluid-structure interaction problem on a vessel of double length.

\section{Conclusions}

In this work we have shown that the coupling between a 3D fluid-structure model and a $1 \mathrm{D}$ reduced model is an effective way to greatly reduce spurious reflections of the pressure waves on the artificial boundaries. We have proposed several strategies for coupling the two models. We have considered solution algorithms based on sub-domains iterations and we have derived an energy estimate for the sub-problems thus obtained.

Acknowledgements: The authors wish to thank Dr. Alessandro Veneziani for many fruitful discussions. This work has been supported by the Swiss National Science Foundation, Project N. 21-54139.98 and by MURST Cofin. 1998, "Advanced Numerical Methods for Scientific Computing".

\section{References}

[1] P. G. Ciarlet. Introduction to linear shell theory. Gauthier-Villars, Paris, 1998.

[2] G.R. Cokelet. The rheology and tube flow of blood. In R. Skalak and S. Chen, editors, Handbook of Bioengineering. McGraw-Hill, 1987.

[3] L. Formaggia, J.F. Gerbeau, F. Nobile, and A. Quarteroni. Boundary treatment for NavierStokes equations by Lagrange Multipliers. in preparation.

[4] L. Formaggia, F. Nobile, A. Quarteroni, and A. Veneziani. Multiscale Modelling of the Circolatory System: a preliminary analysis. to appear in Comp. Vis. Science, 1999.

[5] E. Godlewski and P.-A. Raviart. Numerical approximation of hyperbolic systems of conservation laws, volume 118 of Applied Mathematical Sciences. Springer, New York, 1996.

[6] K. Hayashi, K. Handa, S. Nagasawa, and A. Okumura. Stiffness and elastic behaviour of human intracranial and extracranial arteries. J. Biomech., 13:175-184, 1980.

[7] J.G. Heywood, R. Rannacher, and S. Turek. Artificial boundaries and flux and pressure conditions for the incompressible Navier-Stokes equations. International Journal for Numerical Methods in Fluids, 22:325-352, 1996. 
[8] T.J. Hughes. The finite element method, linear static and dynamic finite element analysis. Prentice-Hall, Englewood Cliffs, 1987.

[9] G.L. Langewouters, K.H. Wesseling, and W.J.A. Goedhard. The elastic properties of 45 human thoracic and 20 abdominal aortas in vitro and the parameters of a new model. $J$. Biomech., 17:425-435, 1984.

[10] D.A. McDonald. Blood Flow in Arteries. Edward Arnold Ltd. - London, 1990. Third edition edited by W.W. Nichols and M.F. O' Rourke.

[11] F. Nobile. Fluid-structure interaction problems in hemodynamics. Master's thesis, Technical University of Milan, 1998. (in italian).

[12] K. Perktold, M. Resch, and H. Florian. Pulsatile non-Newtonian flow characteristics in a three-dimensional human carotid bifurcation model. ASME J. of Biomech. Eng., 113:463475, 1991.

[13] A. Quarteroni, M. Tuveri, and A. Veneziani. Computational vascular fluid dynamics: problems, models and methods. to appear in Comp. Vis. Science, 1998.

[14] A. Quarteroni and A. Valli. Numerical Approximation of Partial Differential Equations. Springer Verlag Series in Computational Mathematics n.23, 1994.

[15] H. Reismann. Elastic plates: theory and application. Wiley, New York, 1988.

[16] J.C. Simo and D.D. Fox. On a stress resultant geometrically exact shell model, Part I: formulation and optimal parametrization. Comput. Meths. Appl. Mech. Engrg., 72:267-304, 1989.

[17] J.C. Simo, D.D. Fox, and M.S. Rifai. On a stress resultant geometrically exact shell model, Part II: the linear theory; computational aspects. Comput. Meths. Appl. Mech. Engrg., 73:53$92,1989$.

[18] J.C. Simo, D.D. Fox, and M.S. Rifai. On a stress resultant geometrically exact shell model, Part III: computational aspects of the nonlinear theory. Comput. Meths. Appl. Mech. Engrg., 79:21-70, 1989.

[19] A. Tozeren. Elastic properties of arteries and their influence on the cardiovascular system. ASME J. Biomech. Eng., 106:182-185, 1984.

[20] A. Veneziani. Mathematical and Numerical Modelling of Blood Flow Problems. PhD thesis, University of Milan, 1998.

[21] X.Y. Xu, M.W. Collins, and C.J.H. Jones. Flow studies in canine aortas. ASME J. of Biomech. Eng., 114(11):504-511, 1992. 


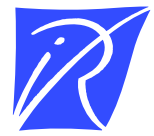

Unité de recherche INRIA Rocquencourt Domaine de Voluceau - Rocquencourt - BP 105 - 78153 Le Chesnay Cedex (France)

Unité de recherche INRIA Lorraine : LORIA, Technopôle de Nancy-Brabois - Campus scientifique 615, rue du Jardin Botanique - BP 101 - 54602 Villers-lès-Nancy Cedex (France)

Unité de recherche INRIA Rennes : IRISA, Campus universitaire de Beaulieu - 35042 Rennes Cedex (France)

Unité de recherche INRIA Rhône-Alpes : 655, avenue de l'Europe - 38330 Montbonnot-St-Martin (France)

Unité de recherche INRIA Sophia Antipolis : 2004, route des Lucioles - BP 93 - 06902 Sophia Antipolis Cedex (France)

INRIA - Domaine de Voluceau - Rocquencourt, BP 105 - 78153 Le Chesnay Cedex (France)

http://www.inria.fr

ISSN 0249-6399 